\title{
Risk-Cost Optimization of Buried Pipelines Using Subset Simulation
}

\author{
Lutfor Rahman Khan ${ }^{1}$ and Kong Fah Tee ${ }^{2}$
}

\begin{abstract}
On the basis of time-dependent reliability analysis, a computational framework called subset simulation (SS) has been applied for risk-cost optimization of flexible underground pipeline networks. SS can provide better resolution for rare failure events that are commonly encountered in pipeline engineering applications. Attention in this work is devoted to scrutinize the robustness of SS in risk-cost optimization of pipelines. SS is first employed to estimate the reliability of flexible underground pipes subjected to externally applied loading and material corrosion. Then SS is extended to determine the intervention year for maintenance and to identify the most appropriate renewal solution and renewal priority by minimizing the risk of failure and whole life-cycle cost. The efficiency of SS compared to genetic algorithm has been demonstrated by numerical studies with a view to prevent unexpected failure of flexible pipes at minimal cost by prioritizing maintenance based on failure severity and system reliability. This paper shows that SS is a more robust method in the decision-making process of reliabilitybased management for underground pipeline networks.
\end{abstract}

Author keywords: Optimization; Subset simulation; Genetic algorithm; Markov chain Monte Carlo; Reliability; Pipeline network.

\section{Introduction}

The reliability of a system is the probability of performing its required functions under stated conditions for a specified period of time. In engineering reliability analysis, it is calculated based on specified probabilistic modeling of the underlying uncertainties or random variables, which is called stochastic algorithm (Li 2011; Schueller and Pradlwarter 2007; Fang et al. 2014). Methods of reliability analysis such as the first-order reliability method (FORM), second-order reliability method (SORM), point estimate method (PEM), Monte Carlo simulation (MCS), gamma process, probability density evolution method (PDEM), etc. are available in literature (Sivakumar Babu and Srivastava 2010; Mahmoodian et al. 2012; Fang et al. 2013; Tee and Khan 2014; Tee et al. 2015). As failure is an exception rather than the rule in properly designed systems, therefore, an engineering reliability analysis involves a rare event simulation. Finding the optimum, on the other hand, involves simulating the extreme events that also can be considered as rare occasions in the design variable space. Thus, if stochastic algorithms are adopted, the objective function is evaluated at the optimum random points in the design variable space.

Most of the decisions that are required during the processes of maintenance and management of aging civil infrastructure are made under conditions of uncertainty. Therefore, such methodologies have to be reliability-based. Recent advances in stochastic optimization have contributed to the development of powerful algorithms for life-cycle cost (LCC) optimization of infrastructure systems under uncertainty. Reliability-based maintenance optimization approaches have been applied in different engineering

\footnotetext{
${ }^{1}$ Geotechnical Engineer, Rail and Ground Engineering, Jacobs Engineering, 1180 Eskdale Rd., Wokingham RG41 5TU, U.K.

${ }^{2}$ Senior Lecturer in Infrastructure Engineering, Dept. of Engineering Science, Univ. of Greenwich, Central Ave., Chatham Maritime, Kent ME4 4TB, U.K. (corresponding author). E-mail: K.F.Tee@gre.ac.uk
}

projects, such as bridges, buildings, pipelines, offshore structures, mechanical components, etc. (Saydam and Frangopol 2015; Beaurepaire et al. 2012; Chen et al. 2013; Tolentino and Ruiz 2013; Tee et al. 2014a). Development of reliability-based management of inspection, monitoring, maintenance, and repair of various types of offshore structures has been described, with a focus on management of hull damage due to crack growth and corrosion (Moan 2005). An existing reinforced-concrete T-beam bridge located in Colorado has been investigated using a time-variant series system reliability approach (Enright and Frangopol 1998). The results can be used to better predict the service life of deteriorating reinforced concrete bridges, and to develop optimal lifetime reliability-based maintenance strategies for these bridges.

Due to the increased safety requirements, the goal of introducing effective optimization for underground pipelines is very challenging because it is difficult to have an algorithm that performs in a uniformly efficient manner for all problems. Several countries have developed or initiated the development of pipe management systems to optimize the maintenance cost of deteriorated pipe structures. Over the last decade, reliability-based optimal design of buried pipe distribution systems has been studied by several researchers and this has resulted in the development of a number of reliability models and the application of optimization techniques (Rahman and Vanier 2004). Different optimization approaches have been implemented in different buried pipe management systems ranging from simplified economic models to advanced Markovian decision processes (Lounis 2006).

Nevertheless, the design of these models is based on future predefined and perfectly known working conditions, a premise that directly impacts the optimization process. The imposed scenario may perform badly if the reality turns out significantly different. In the context of a proactive attitude toward these risks, it is important to consider these aspects at the beginning of the design phase. Arranging these activities in a proper time scale is also a difficult task. Different pipeline projects require different timetables to complete. A shorter project duration may lead to higher direct costs as more skilled laborers and resources are needed for completion in a short time. In contrast, a longer project duration may lead to lower direct and indirect costs. In such situations, it is 
important to study the trade-off between completion time, the risk involved in each resource option, and the cost of the project (Halfawy et al. 2008; Tee and Li 2011). Trading off between these conflicting aspects of a project is a challenging job and, in such cases, planners are faced with numerous possible combinations for project delivery. If durations of the activities are impacted, the cost may show an increasing trend due to more resources being allocated for their rapid accomplishment (Ambrose et al. 2008).

It is commonly believed that there is no single universal method thath is capable of solving all kinds of optimization problems efficiently because each method has its own definition and limitations. A common feature of most stochastic optimization algorithms is that they are developed based on the observations of random phenomena in nature. Hence, the random sampling and/or random manipulation play an important role in the implementation of these algorithms. These random features provide the possibility of jumping out of local optima. Various sophisticated numerical techniques based on gradient information have been well documented (Ravindran et al. 2006), but most of them are vulnerable to convergence into a local optimum due to nonlinear, multimodal, or even discontinuous objective functions. Stochastic optimization algorithms are commonly used for solving global and local optimization problems. Many are based on probabilistic assumptions on the design variables and the objective functions. Among stochastic optimization algorithms, simulated annealing (SA) and genetic algorithm (GA) have been most successful (Spall 2003). However, these are computationally expensive traditional algorithms. In addition, there is no absolute assurance that these methods will find a global optimum.

To overcome the above limitations, a stochastic optimization algorithm, subset simulation (SS), has been applied in this paper, which is more robust and easy to implement for solving nonlinear and multimodal optimization problems. SS is a relatively new method and has not yet been applied in optimization for pipe maintenance. SS, which is originally a reliability analysis method, can be implemented to solve risk-cost optimization problems for underground pipelines by introducing artificial probabilistic assumptions on design variables (Au and Beck 2001, 2003; Au et al. 2007; Li 2011; Tee et al. 2014b). The first part of the proposed method is a reliability analysis approach to calculate the probability of failure based on corrosion-induced failure modes, whereas the second part is a risk-cost optimization approach to determine the intervention year for maintenance from the minimization of the total LCC. The calculated probability of failure from the first part will be used as an input to the second part. Both the reliability analysis and risk-cost optimization are performed using SS. Appropriate renewal methods and renewal priority can also be obtained.

The basic idea is to deal with the optimization problems in the context of reliability analysis. The searching process of an optimization problem is equivalent to exploring the process of the tail distribution in a reliability problem. Based on the concept of SS, this artificial reliability problem is decomposed into a series of conditional probability problems. The modified Metropolis-Hastings algorithm (Au and Beck 2001; Au et al. 2007) is implemented to generate efficiently the conditional solutions in each simulation level. An ascending sequence of the objective function values is chosen adaptively so that the estimated conditional probabilities are equal to the specified value. When increasing the sequence, the value of the objective function approaches the optimum point.

\section{Basics of SS Optimization}

The analogy between an optimization problem and a reliability problem allows an optimization problem to be solved using the
SS method. Let $h_{\mathrm{opt}}$ be the global maximum of $h$, where $x=$ $x_{\text {opt }}$. By definition of cumulative density function (CDF), a CDF curve is monotonic, nondecreasing, and right-continuous and its value at $h=h_{\text {opt }}$ is unity. A reliability problem including classical and stochastic ones can be employed to perform as an optimization algorithm (process). However, solving optimization problems by reliability methods is still in its infancy compared to other available methods, such as GA, finite state machine (FSM), ant colony optimization algorithm (ACOA), shuffled frog leaping algorithm (SFLA), etc., due to lack of research and applications in practical problems ( $\mathrm{Li}$ and $\mathrm{Au} 2010$ ).

The basic difference between a reliability and an optimization problem is that the aim of a reliability analysis is to evaluate the probability of an event, while the aim of an optimization problem is to locate a point or region where the objective function is minimized or maximized, i.e., taking extreme values. One can therefore treat the optimization problem as locating a rare event, because an extreme event is also a special case of a rare event. Based on these observations, an optimization problem can be converted to a reliability problem, which makes it possible to use a reliability method for solving an optimization problem. To view the optimization problem in the framework of reliability analysis, the design variables are artificially considered to be random ( $\mathrm{Li}$ and $\mathrm{Au}$ 2010). This induces the objective function $h$ to $P_{F}$.

Consider an optimization problem given by

$$
\operatorname{Max} h(x), \quad \text { such that } x \in \Omega
$$

where $h: \Omega \subset R^{n} \rightarrow R=$ real valued function; $x=$ design variable vector; and $\Omega=$ closed and bounded set. The maximum $\left(x_{\mathrm{opt}}, h_{\mathrm{opt}}\right)$ is the point such that

$$
h_{\mathrm{opt}}=h\left(x_{\mathrm{opt}}\right) \geq h(x)
$$

In Eq. (1), only one variable $x$ is involved, and $h$ is a function of $x$. The optimization problem is defined as finding the maximal value of $h$, i.e., $\max h(x)$.

On the other hand, a reliability problem can be defined to estimate the probability of $h$ exceeding a given threshold $h_{0}$; therefore, the failure probability can be estimated as shown in Eq. (2):

$$
P_{f}=P\left[h(x) \geq h_{0}\right]
$$

The reliability problem is often considered a rare event simulation problem because a very small probability of failure is involved in practice. Geometrically, the region of $h>h_{0}$ in a reliability problem is broader than that of maximum points in an optimization problem. In other words, the region needing to be found in an optimization problem is a reduced region in a reliability problem. The attention here is on the point or region where the objective function attains the largest value, which can be a challenging problem when the objective function has many local optimums or when the dimension of the design variable space is large (Li 2011). Recall that the artificial reliability problem is not to compute the failure probability but to search for the corresponding point $h_{\mathrm{opt}}$ of zero failure probability given by Eq. (2).

After randomizing the design variables, the objective function maps the multidimensional design variable vector into a random variable $h$. This mapping also transforms all points that take the same value onto one point on the nondecreasing CDF curve of the randomized objective function $h$. Hence, the trend trapping in local optimums can be easily avoided ( $\mathrm{Li}$ and $\mathrm{Au} 2010$ ). Based on the aforementioned conventions, it is clear that an artificial reliability problem can be dealt with using an optimization problem in the framework of reliability analysis. Thus, SS can generate samples 
(solutions) that progress toward the maximum in a more efficient way, simultaneously as the rare event region is gradually being populated.

\section{Pipe Failure Modes}

When the residual ultimate strength of a buried pipeline is exceeded, breakage becomes imminent and the overall reliability of the pipe is reduced. The critical failure modes are those contributing significantly to the reliability of the system at the chosen level. The failure criteria adopted here are due to loss of structural strength of pipelines by corrosion through reduction of the pipe wall thickness, which then leads to pipe failure by excessive deflection, buckling, wall thrust, and bending.

\section{Corrosion of Metal Pipes}

Buried pipes are made of plastic, concrete, or metal, e.g., steel, galvanized steel, ductile iron, cast iron, or copper. Plastic pipes tend to be resistant to corrosion. Damage in concrete pipes can be attributed to biogenous sulfuric acid attack (Tee et al. 2011; Alani et al. 2014). On the other hand, metal pipes are susceptible to corrosion. Metal pipe corrosion pitting is a continuous and variable process. The corrosion pit depth can be modeled with respect to time as shown in Eq. (3) (Ahammed and Melchers 1997; Sadiq et al. 2004).

The corrosion pit depth is

$$
D_{T}=k T^{n}
$$

where $D_{T}=$ pit depth; and $T=$ exposure time. The parameters $k$ and $n$ are corrosion empirical constants and depend on pipe materials and surrounding environments. Due to the reduction of wall thickness given by Eq. (3), the moment of inertia of pipe wall per unit length $I$ and the cross-sectional area per unit length $A_{s}$ for thin-walled plain pipe can be defined as follows (Watkins and Anderson 2000; Tee and Khan 2012):

$$
\begin{gathered}
\text { Moment of inertia: } I=\left(t-D_{T}\right)^{3} / 12 \\
\text { Cross-sectional area: } A_{s}=t-D_{T}
\end{gathered}
$$

where $t=$ thickness of pipe wall.

\section{Deflection, Buckling Pressure, Wall Thrust, and Bending Stress}

In this paper, the chosen dominating failure modes of flexible pipes are characterized by corrosion-induced excessive deflection, buckling, wall thrust, and bending stress (Tee et al. 2013). These dominating failure modes are discussed briefly as follows.

A buried pipe tends to deflect under the effects of earth and live loads. The performance of a flexible pipe in respect to its ability to support the load is typically assessed by measuring the deflection from its initial shape. Deflection is quantified in terms of the ratio of the horizontal increase in diameter (or vertical decrease in diameter) to the original pipe diameter. Rigid pipe is sometimes classified as pipe that cannot deflect more than $2 \%$ of its internal diameter without significant structural distress, such as cracking (Hancor 2009). Flexible pipe takes advantage of its ability to move, or deflect under loads without structural damage. The actual deflection, $\Delta_{y}$, can be calculated as shown in Eq. (6) (Watkins and Anderson 2000). The critical, or allowable, deflection for flexible pipe, $\Delta y_{c r}$, is normally determined as $5 \%$ of the inside diameter of a pipe (Gabriel 2011):

$$
\Delta_{y}=\frac{K_{b}\left(D_{L} W_{c}+P_{s}\right) D}{\left(\frac{8 E I}{D^{3}}+0.061 E^{\prime}\right)}
$$

where $K_{b}=$ deflection coefficient; $D_{L}=$ deflection lag factor; $D=$ mean diameter; $E=$ modulus of elasticity of pipe material; $E^{\prime}=$ modulus of soil reaction; $W_{c}=$ soil load; and $P_{s}=$ live load.

External loadings from soil pressure or external hydrostatic pressure can cause inward deformation known as wall buckling. Buckling is a premature failure in which the pipe is not able to maintain its initial circular shape and the structure becomes unstable at a stress level that is well below the yield strength of the structural material (Sivakumar Babu and Srivastava 2010). The actual buckling pressure, $p$, and the critical buckling pressure, $p_{c r}$, can be calculated from Eqs. (7) and (8), respectively (AWWA 1999):

$$
p=R_{w} \gamma_{s}+\gamma_{w} H_{w}+P_{s}
$$

$$
p_{c r}=\sqrt{\left(32 R_{w} B^{\prime} E_{s} \frac{E I}{D^{3}}\right)}
$$

where $R_{w}=$ water buoyancy factor; $\gamma_{s}$ and $\gamma_{w}=$ unit weight of soil and water, respectively; $H_{w}=$ height of groundwater above the pipe; $B^{\prime}=$ empirical coefficient of elastic support; and $E_{s}=$ soil modulus.

If the buried depth is not enough then the pipe wall can crush (wall thrust) due to earth and surface loading. The allowable or critical thrust must be equal to or greater than the actual thrust in order for the pipe to remain structurally stable. The critical and actual wall thrust can be estimated as shown in Eqs. (9) and (10), respectively (Hancor 2009):

$$
\begin{gathered}
T_{c r}=F_{y} A_{s} \phi_{p} \\
T_{a}=\left(W_{A}+P_{S} C_{L}+P_{w}\right)\left(D_{0} / 2\right)
\end{gathered}
$$

where $F_{y}=$ minimum tensile strength of pipe; $\phi_{p}=$ capacity modification factor for pipe; $C_{L}=$ live load distribution coefficient; $W_{A}=$ soil arch load; $P_{w}=$ hydrostatic pressure and $D_{o}=$ outside diameter of pipe.

If a pipe is part of a carrying structure, the elastic limit may be an obvious choice as the design limit. A pipe subjected to increasing pure bending will fail as a result of increased ovalization of the cross section and reduced slope in the stress-strain curve. The actual bending stress can be measured from Eq. (11). The allowable bending stress is the long-term tensile strength of pipe material:

$$
\sigma_{b}=2 D_{f} E \Delta_{y} y_{0} / D^{2}
$$

where $D_{f}=$ shape factor; and $y_{0}=$ distance from centroid of pipe wall to the furthest surface of the pipe.

\section{Methodology}

The SS optimization is combined with the total life-cycle cost [Eq. (15)] as the objective function, which will be explained in the next section. The procedure of the SS optimization algorithm is presented as follows. First, select the distributional parameters for design variables. In the original optimization problem, each design variable is a random variable with an artificial probability density function (PDF) $f(x)$. If there are $n$ numbers of design variables denoted as $x=\left\{x_{1}, x_{2}, \ldots, x_{n}\right\}^{T}$, then the corresponding PDFs of these design variables are denoted as $f_{1}\left(x_{1}\right), f_{2}\left(x_{2}\right), \ldots, f_{n}\left(x_{n}\right)$. It is assumed that the random variables are statistically independent; 
hence, the joint PDF is the product of the PDFs of the random variables. Therefore, the joint PDF of all design parameters can be expressed as shown in Eq. (12) (Au and Beck 2003; Ching et al. 2005; Li 2011):

$$
f(x)=\prod_{i=1}^{n} f_{i}\left(x_{i}\right)
$$

Next, generate $N$ independent and identically distributed samples $\left\{x_{1}, x_{2}, \ldots, x_{n}\right\}$ by direct Monte Carlo simulation according to the artificial distributions. Each sample $x_{i}(i=1,2, \ldots, n)$ has $n$ components, i.e., $x_{i}=\left\{x_{i}^{(1)}, x_{2}^{(2)}, \ldots, x_{i}^{(n)}\right\}$, where $x_{i}^{(j)}\{j=$ $1,2, \ldots, n\}$ are generated from $f_{j}\left(x_{j}\right)(j=1,2, \ldots, n)$. Calculate the constraint fitness function values (if any) and the objective function values of the samples and then sort them according to the double-criterion ranking algorithm. The first ranking based on the constraint fitness function is designed to search the feasible domain, while the second ranking based on objective function searches for the optimal solution.

The modified Metropolis-Hastings (MMH) algorithm is employed for generating samples conditional on the intermediate event. Let $f_{i}^{*}\left(\xi_{i} \mid x_{i}\right), i=1,2, \ldots, n$ be a series of PDFs that depend on $x_{i}$. In order to generate the next Markov chain sample $x_{k+1}=\left\{x_{k+1}^{(1)}, x_{k+2}^{(2)}, \ldots, x_{k+1}^{(n)}\right\}^{T}$ from the current sample $x_{k}=$ $\left\{x_{k}^{(1)}, x_{k}^{(2)}, \ldots, x_{k}^{(n)}\right\}^{T}$ conditional on an event $F_{j}$, the MMH algorithm is presented as follows ( $\mathrm{Au}$ and Beck 2001; Li 2011):

1. Generate a candidate state $\xi_{k+1}=\left\{\xi_{k+1}^{(1)}, \xi_{k+2}^{(2)}, \ldots, \xi_{k+1}^{(\dot{n})}\right\}^{T}$ from the proposal PDFs

$$
f_{i}^{*}\left(\xi_{i} \mid x_{i}\right), \quad i=1,2, \ldots, n
$$

For each component $i=1,2, \ldots, n$

a. Generate a precandidate component $\xi_{k+1}^{(1)}$ from $f_{i}^{*}\left(\xi_{i} \mid x_{i}\right)$

$b$. Compute the acceptance ratio as

$$
r_{k+1}^{i}=\frac{f_{i}\left(\xi_{k+1}^{(i)}\right) f_{i}^{*}\left(x_{k}^{i} \mid \xi_{k+1}^{(i)}\right)}{f_{i}\left(x_{k}^{(i)}\right) f_{i}^{*}\left(\xi_{k+1}^{(i)} \mid x_{k}^{i}\right)}
$$

c. Set the $i$ th component of $\xi_{k+1}$ according to

$$
\xi_{k+1}^{(i)}=\left\{\begin{array}{l}
\xi_{k+1}^{(i)} \text { with probability } \min \left(1, r_{k+1}(i)\right) \\
x_{k}^{(i)} \text { with probability } 1-\min \left(1, r_{k+1}^{(i)}\right)
\end{array}\right.
$$

2. When $\xi_{k+1} \neq x_{k}$ perform an evaluation of the objective function $h\left(\xi_{k+1}\right)$. If $\xi_{k+1} \in F_{j}$, accept it as the next state, otherwise reject it and take the current state as the next one, i.e., set $x_{k+1}=x_{k}$. $\mathrm{Au}$ et al. (2007) have proved that the next sample will be distributed as the current one is, and, hence, is the stationary distribution of the Markov chain.

In each simulation level, a Markov chain can be generated with the same conditioning. Because the initial samples obey the conditional distribution, all these Markov chains are automatically in a stationary state and samples in these Markov chains distribute according to the conditional distribution. If the number of samples in each level is a constant number, then the length of each Markov chain will be $1 / p_{k-1}(k=2, \ldots, m)$ where $p_{k-1}$ is the level probability in the last simulation level and $m$ is the total simulation levels. Evaluate and sort the objective function values of new generated samples. Then determine the $N\left(1-p_{k}\right)$ th percentile $h_{k, N\left(1-p_{k}\right)}$ from the ascending sequence of $\left\{h_{k, l}: l=1, \ldots, N\right\}$ so that the probability of the conditional event is satisfied, as shown in Eq. (13) (Li 2011):

$$
P\left(F_{\mathrm{k}} \mid F_{k-1}\right)=P\left[h(x) \geq h_{k, N\left(1-p_{k}\right)} \mid F_{k-1}\right] \approx p_{k}
$$

These samples with their objective function values larger than $h_{k, N\left(1-p_{k}\right)}$ are chosen to provide seeds for the sampling operation in the next simulation level. The procedure is repeated until a stop criterion is met or the computational budget of the objective function evaluations is exhausted.

Each new simulation level is an improvement on the previous one that went before, which is realized by the level probability $p_{k}$, as shown in Eq. (13). The level probability $p_{k}$ is used to select a subpopulation that provides the sampling seeds for the next simulation level. It controls the efficiency of calculation and the choice of level probability is a trade-off between the number of samples required in each simulation level and the number of simulation levels required to reach the target failure region. Au et al. (2007) suggested that $p_{k}$ takes a constant value of 0.1 to 0.2 in a real reliability problem. In SS optimization, the level probability is a control parameter that regulates the convergence of the optimization process. If a very small number is used, the algorithm would have a low probability of reaching a global optimum. Therefore, the level probability must be large enough to permit the locally developed Markov chain samples to move out of a local optimum in favor of finding a global optimum, especially in the early simulation level. However, a large value would increase the number of simulation levels.

The total number of samples is equal to $N+\sum_{k=2}^{m}\left(1-p_{k}\right) N$. It is observed that a large value of $N$ tends to increase the number of objective function evaluation, which is a time-consuming process. On the other hand, a small value of $N$ may cause the generated samples not to cover the searching space well and thus lead to a fail in finding a good optimum solution. Therefore, in practice, this number should not be too large or too small. More details on SS optimization can be found in $\mathrm{Li}$ (2011).

\section{Life-Cycle Cost}

For a pipeline, the occurrence of either failure mode will constitute its failure. Therefore, a series system is more appropriate for its assessment of failures. The correlation between the failure modes is estimated and the value is within the range from 0 to 1 . Thus, the probability of failure for a series system $P_{f}$ can be estimated as follows (Fetz and Tonon 2008):

$$
\operatorname{Max}\left[P_{f, i}\right] \leq P_{f} \leq 1-\prod_{i=1}^{r}\left[1-P_{f, i}\right]
$$

where $P_{f, i}=$ probability of failure due to $i$ th failure mode of pipe; and $r=$ number of failure modes considered in the system.

In this paper, the life-cycle cost (LCC) consists of initial cost or installation cost, maintenance cost, and failure risk cost (Ambrose et al. 2008). The total life-cycle cost $C_{\mathrm{LCC}}(T)$ can be presented as follows (Hinow et al. 2008):

$$
C_{\mathrm{LCC}}(T)=C_{A}+\sum_{j=1}^{T} C_{M}(j)+\sum_{j=1}^{T} C_{f}(j) \times P_{f, j}
$$

where $C_{A}=$ capital cost; $C_{M}=$ maintenance cost; $C_{f}=$ failure cost, and $j=1,2,3, \ldots, T$ year.

In this paper, the problem of identifying the optimal intervention year is transformed into minimization of total LCC [Eq. (15)] using SS. The cost terms in the right-hand side of Eq. (15) are the costs in the year they actually occur. The $(1+r)^{T}$ factor is used to convert the cost into its present value discounted by the discount rate of $r$, 
Table 1. Failure Impact Rating

\begin{tabular}{lc}
\hline Weighted impact factor, $I_{w}$ & Failure impact rating, $R_{\text {imp }}$ \\
\hline 1.00 & 1 \\
$1.01-1.60$ & 2 \\
$1.61-2.20$ & 3 \\
$2.21-2.80$ & 4 \\
$>2.81$ & 5 \\
\hline
\end{tabular}

Table 2. Possibility of Soil Loss Based on Soil Type and Groundwater Level

\begin{tabular}{lccc}
\hline & \multicolumn{3}{c}{ Groundwater level } \\
\cline { 2 - 4 } Soil type & Below & Same line & Above \\
sewer & with sewer & sewer \\
\hline Clay & Low & Medium & High \\
Gravels and low-plasticity clay & Low & Medium & High \\
Silt and sand & High & High & High \\
\hline
\end{tabular}

for the $T$ year period. The discount rate depends on the prevailing interest rate and the depreciation of the currency or inflation rate.

\section{Maintenance Strategy}

The criterion used for pipe renewal is the degree of impact of an underground pipeline failure. The impact assessment ranks the pipe segments in unit length in terms of six major factors: location, embedment soil, burial depth, pipe size, functionality, and seismic zone. The assessment generates a ranking of impact for the underground pipeline system. Each of the six factors is assigned a degree of impact defined by low, medium, or high (McDonald and Zhao 2001). A weighted impact rating $\left(I_{w}\right)$ formula is used to combine the influence of each of the six factors for each pipe segment within the system as follows:

$$
I_{w}=0.2 f_{l}+0.16\left(f_{s}+f_{z}+f_{d}+f_{f}+f_{q}\right)
$$

where $f_{l}=$ location factor; $f_{s}=$ embedment soil factor; $f_{z}=$ size factor; $f_{d}=$ burial depth factor; $f_{f}=$ underground pipeline function factor; and $f_{q}=$ seismic factor. Although these factors do not change dramatically from year to year, periodic updating may be necessary. The failure impact rating $R_{\text {imp }}$ can be assessed based on Table 1 with respect to $I_{w}$ values (McDonald and Zhao 2001). For all of the factors listed, the low value is 1 and high value is 3 . The medium degree of impact falls between the high and low extremes and is assigned a value of 1.5 .

Once the weighted impact rating is determined for individual pipe segments, the impact assessment can then be used in a number of ways in the decision-making process. The impact ratings can be used in combination with the underground pipeline condition, which is called the condition index, or the mean structural pipe grade to prioritize rehabilitation or replacement work and the future inspection frequencies. The maintenance strategy can be implemented by identifying applicable renewal categories based on the underground pipeline condition index. The purpose of the condition index is to objectively rate or scale the current condition of buried pipes based on several physical, environmental, and operational factors, which provide the basic terminology and framework. The mean structural pipe grade or structural condition index $(C I)$ for underground pipeline can be calculated from the regression model as follows (WRC 2001):

$$
C I=0.0003 T^{2}-0.0003 T+1
$$

where $T=$ age of underground pipeline (in years), which corresponds to the intervention year obtained from the risk-cost optimization.

Buried pipeline renewal methods can be grouped into four main categories: replacement, structural, semistructural, and nonstructural lining methods (Khan et al. 2013). Structural liners are defined as being capable of carrying hydrostatic, soil, and live loads on their own. Structural liners are expected to be independent, i.e., bonding with original underground pipeline is not required. Semistructural liners are designed to withstand hydrostatic pressure or perform as a composite with the existing pipelines. Semistructural liners could be designed as interactive or independent. Semistructural liners typically are used for nongravity pipeline systems. Nonstructural liners are used mainly to improve flow, resist corrosion, or to seal minor cracks in gravity pipelines.

The possibility of surrounding soil loss, a very important parameter to assess the renewal process, is determined on a high, medium, or low scale according to the soil type and groundwater level, as shown in Table 2 (Newton and Vanier 2006). The renewal methods are selected based on detailed analysis of possible defects, as indicated by the condition index and the possible scenarios of soil loss, as shown in Table 3 (WRC 2001). For example, an underground pipeline with Condition Index 3 and a high possibility of soil loss will need replacement or the use of a structural liner to carry loads and stabilize deformation. At a minimum, a semistructural liner that can withstand hydrostatic pressure is required. On the other hand, the renewal priorities are decided based on $C I$, $I_{w}$, and $R_{\text {imp }}$ values as shown in Table 4 . For the pipe segments with the same physical condition index/rating, those with higher impact ratings would be considered first for rehabilitation.

\section{Numerical Example}

An underground pipeline network under a roadway subjected to heavy load operating conditions, passing under commercial and residential areas, is taken as a numerical example to validate the proposed risk-cost optimization management strategy using SS. The underground pipeline network, which was constructed in 1940 , consists of approximately $789 \mathrm{~km}$ of sanitary flexible buried metal pipelines. The pipes in the network are medium-sized steel and ductile iron pipes. The whole network is divided into six segments of pipeline, namely, A to F. The pipe material, location, and

Table 3. Selection of Renewal Categories Based on Condition Index and Soil Loss Possibility

\begin{tabular}{lccc}
\hline & & Possibility of soil loss & \\
\cline { 2 - 4 } Condition index & Low & Medium & High \\
\hline 2 & Nonstructural or semistructural & Nonstructural or semistructural & Semistructural, structural, or replacement \\
3 & Nonstructural or semistructural & Semistructural or structural & Semistructural, structural, or replacement \\
4 and 5 & Structural or replacement & Structural or replacement & Structural or replacement \\
\hline
\end{tabular}


Table 4. Renewal Priority

\begin{tabular}{|c|c|c|c|}
\hline $\begin{array}{l}\text { Structural } \\
\text { condition } \\
\text { index }\end{array}$ & Implication & $\begin{array}{c}\text { Failure impact } \\
\text { rating }\left(R_{\text {imp }}\right)\end{array}$ & $\begin{array}{l}\text { Renewal } \\
\text { priority }\end{array}$ \\
\hline 5 & Failed or failure imminent & 1 to 5 & Immediate \\
\hline \multirow[t]{2}{*}{4} & Very poor condition & 5 & Immediate \\
\hline & High structural risk & 1 to 4 & High \\
\hline \multirow[t]{2}{*}{3} & Poor condition & 4 to 5 & Medium \\
\hline & Moderate structural risk & 1 to 3 & Low \\
\hline 2 & $\begin{array}{l}\text { Fair condition/minimal } \\
\text { structural risk }\end{array}$ & 1 to 5 & Low \\
\hline 1 or 0 & Good or excellent condition & 1 to 5 & Not requirec \\
\hline
\end{tabular}

soil parameters of each segment are listed in Table 5. The whole network was constructed above the groundwater table.

It is presumed that the whole underground pipeline network is located in a high seismic vulnerable zone area. The network is subjected to corrosion and its corrosion is presumed to be uniform over the pipe sections. The corrosion rate is modeled using Eq. (3). There are nine random variables (elastic modulus of pipe, soil modulus, soil density, live load, deflection coefficient, corrosion coefficients, pipe wall thickness, and height of the backfill) for which the mean and coefficient of variation are listed in Table 6. The data are obtained from Ahammed and Melchers (1997), Sadiq et al. (2004), and Sivakumar Babu and Rao (2005).

The capital cost, maintenance cost, and failure consequence cost (future values) are presented in Table 7 on a yearly basis for the whole network from Sections A to F. The cost data are predicted or calibrated based on real case studies on municipal infrastructure investment planning (MIIP) in Canada (Rahman and Vanier 2004), a report by Melbourne Water (owned by the Victorian government) (2012), and Davis et al. (2008). A typical discount rate of 5\% is considered in this paper. In this paper, LCC is used as an objective function, which includes initial construction cost, maintenance cost, and failure cost. All alternatives for maintenance and renewal will be considered. The essence of LCC is that one alternative may have a higher initial cost, but its costs over the asset's life cycle may be lower than those of other alternatives.

\section{Results and Discussion}

The SS optimization process has been developed to solve the riskcost optimization problem of a buried pipeline network in order to validate the proposed method. This numerical example is an unconstrained optimization problem. In this paper, the design variable is the failure probability of the underground pipeline network, which comprises nine random variables as listed in Table 6 , and the total LCC in Eq. (15) is the objective function. To obtain a fair and reliable result, a considerable amount of computational effort and time has been spent to identify the best number of samples required
Table 6. Statistical Properties

\begin{tabular}{lccc}
\hline Symbol description & $\begin{array}{c}\text { Mean } \\
\text { value }\end{array}$ & $\begin{array}{c}\text { Coefficient } \\
\text { of variation } \%\end{array}$ & Distribution \\
\hline $\begin{array}{l}\text { Elastic modulus } \\
\text { of steel pipe }\end{array}$ & $210 \mathrm{GPa}$ & 1.0 & Normal \\
$\begin{array}{l}\text { Elastic modulus of } \\
\text { ductile iron pipe }\end{array}$ & $170 \mathrm{GPa}$ & 1.0 & Normal \\
$\begin{array}{l}\text { Soil modulus, } E_{s} \\
\text { Unit weight of soil, } \gamma\end{array}$ & $2 \mathrm{MPa}$ & 5 & Normal \\
Traffic load (live load), $P_{s}$ & Table 5 & 3.0 & Normal \\
Deflection coefficient, $K_{b}$ & 0.11 & 1.0 & Normal \\
Multiplying constant, $k$ & 0.3 & 10.0 & Normal \\
Exponential constant, $n$ & 0.6 & 5.0 & Normal \\
\hline
\end{tabular}

for achieving a reasonably good solution. The results are presented as follows.

\section{Pipeline Reliability}

First, the probabilities of buried pipe failure due to corrosioninduced excessive deflection, buckling, wall thrust, and bending with respect to time are estimated using the SS method based on the parameters and basic variables given in Tables 5 and 6 . When the thickness of the pipe is reduced due to corrosion, the moment of inertia and the cross-sectional area of the pipe wall are decreased with a resulting reduction in pipe strength, as shown in Eqs. (4) and (5), respectively. All the random variables are considered as normally distributed, except the deflection coefficient, which is lognormally distributed. For the non-normally distributed variable, the Rosenblatt transformation is used to transform it to an equivalent normal variable. The occurrence of either failure mode of the pipe will constitute its failure. Therefore, the probability of failure of the underground pipeline network is determined as a series system using Eq. (14) and the results are shown in Figs. 1-6.

The paper shows that on average the probability of pipe failure at the beginning is close to zero and it remains unchanged until about 45 years of service life. Then it gradually changes as time increases; after 50 years, the probability of failure rises drastically. Upper failure probabilities, as shown in Figs. 1-6 for pipe Sections A to $\mathrm{F}$, have been used for the subsequent risk-cost optimization as a worst-case scenario.

\section{Optimum Renewal Cost, Time, and Priority}

As shown in Eq. (15), the failure risk cost is calculated by multiplying the failure cost with the probability of system failure. Once the probability of system failure has been calculated, the optimal time to repair or replace, as well as the associated life-cycle risk and cost, are obtained from the risk-cost optimization using SS. Table 8 summarizes the results of the SS optimization process for the convergence of total LCC obtained from pipeline risk-cost

Table 5. Pipe Materials and Location Properties

\begin{tabular}{|c|c|c|c|c|c|c|c|c|}
\hline Pipe section & Material & Location & Embedment soil & $\begin{array}{l}\text { Length } \\
(\mathrm{km})\end{array}$ & $\begin{array}{c}\text { Mean } \\
\text { diameter }(\mathrm{mm})\end{array}$ & $\begin{array}{l}\text { Thickness } \\
(\mathrm{mm})\end{array}$ & $\begin{array}{l}\text { Soil height above } \\
\text { pipe invert }(\mathrm{m})\end{array}$ & $\begin{array}{c}\text { Traffic } \\
\text { load }(\mathrm{kPa})\end{array}$ \\
\hline A & Steel & Commercial & Clay & 150 & 500 & 8 & 2.0 & 100 \\
\hline B & Ductile iron & Commercial & Clay & 100 & 600 & 8 & 2.0 & 100 \\
\hline $\mathrm{C}$ & Steel & Residential & Sand & 110 & 600 & 9 & 2.1 & 100 \\
\hline D & Steel & Residential & Sand & 225 & 480 & 7.5 & 2.5 & 90 \\
\hline E & Ductile iron & Residential & Sandy gravel & 85 & 350 & 7 & 2.2 & 100 \\
\hline $\mathrm{F}$ & Ductile iron & Commercial & Sandy gravel & 115 & 500 & 8 & 1.8 & 100 \\
\hline
\end{tabular}


Table 7. Cost Data for Pipe Network

\begin{tabular}{lccc}
\hline Pipe section & Capital cost & Maintenance cost & Failure cost \\
\hline A & $£ 1$ million & $£ 20,000$ & $£ 100$ million \\
B & $£ 50,000$ & $£ 10,000$ & $£ 80$ million \\
C & $£ 70,000$ & $£ 8,000$ & $£ 90$ million \\
D & $£ 1$ million & $£ 15,000$ & $£ 140$ million \\
E & $£ 30,000$ & $£ 8,000$ & $£ 70$ million \\
F & $£ 55,000$ & $£ 7,000$ & $£ 85$ million \\
\hline
\end{tabular}

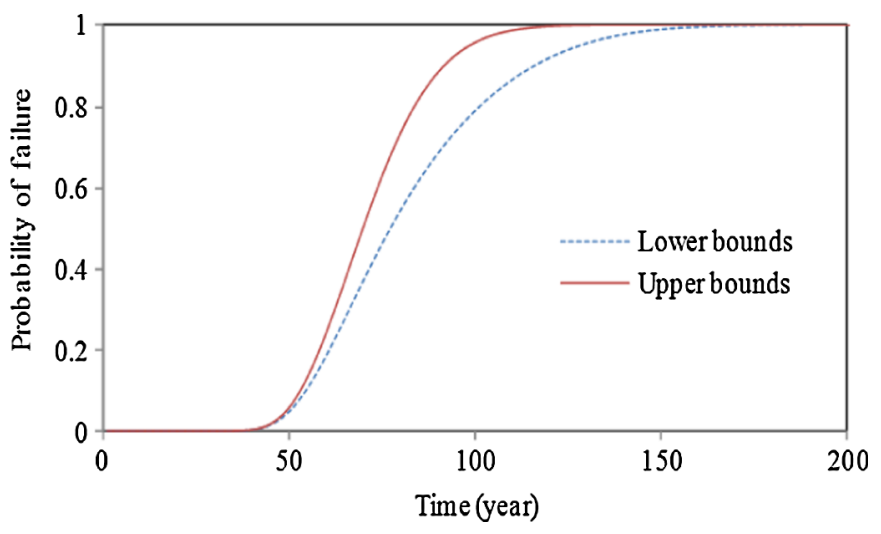

Fig. 1. Probability of failure for pipeline Section A using SS

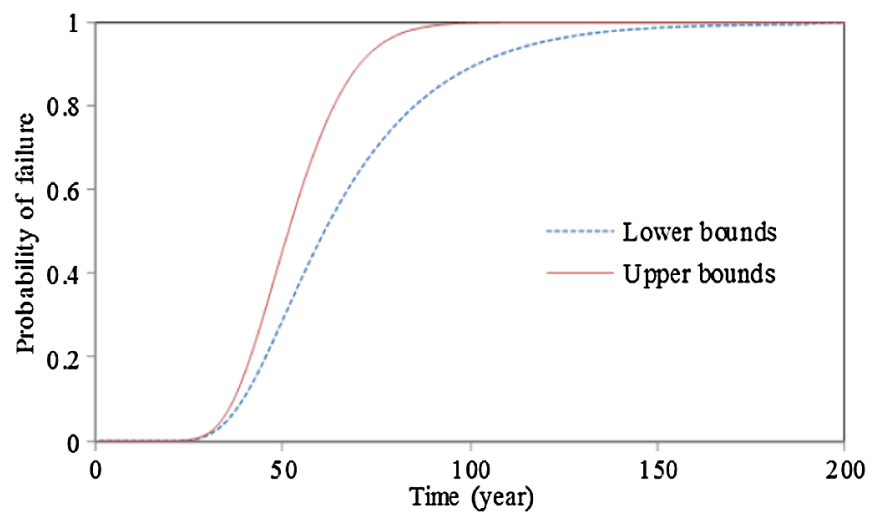

Fig. 2. Probability of failure for pipeline Section B using SS

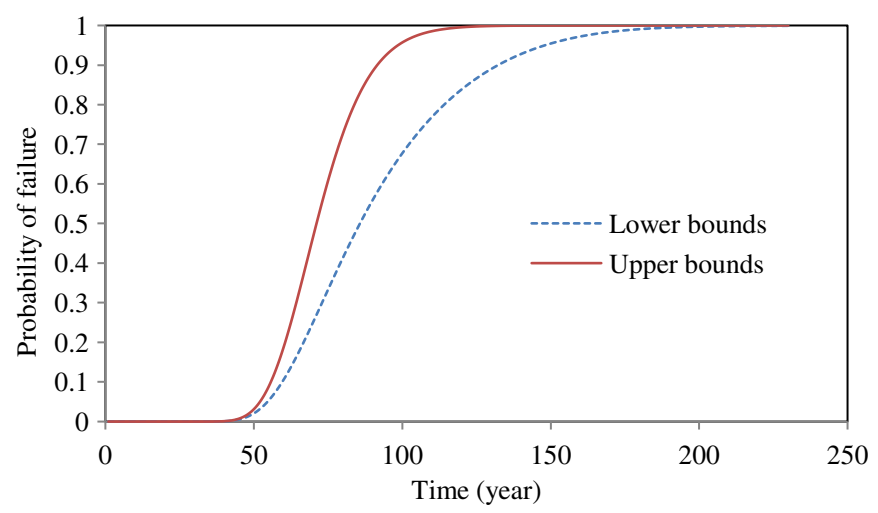

Fig. 3. Probability of failure for pipeline Section C using SS

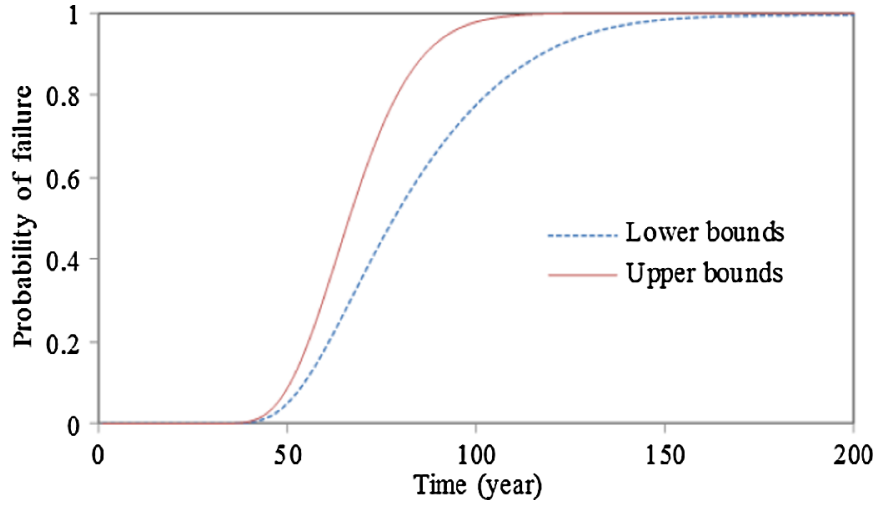

Fig. 4. Probability of failure for pipeline Section D using SS

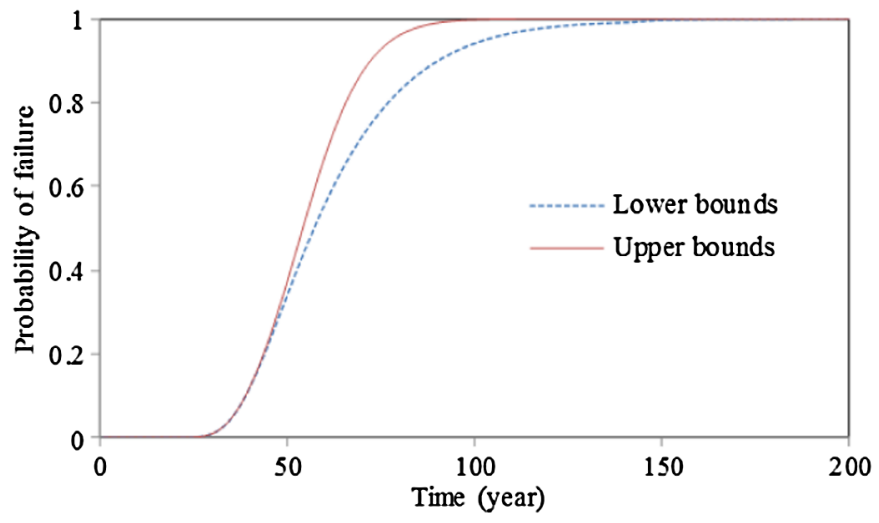

Fig. 5. Probability of failure for pipeline Section E using SS

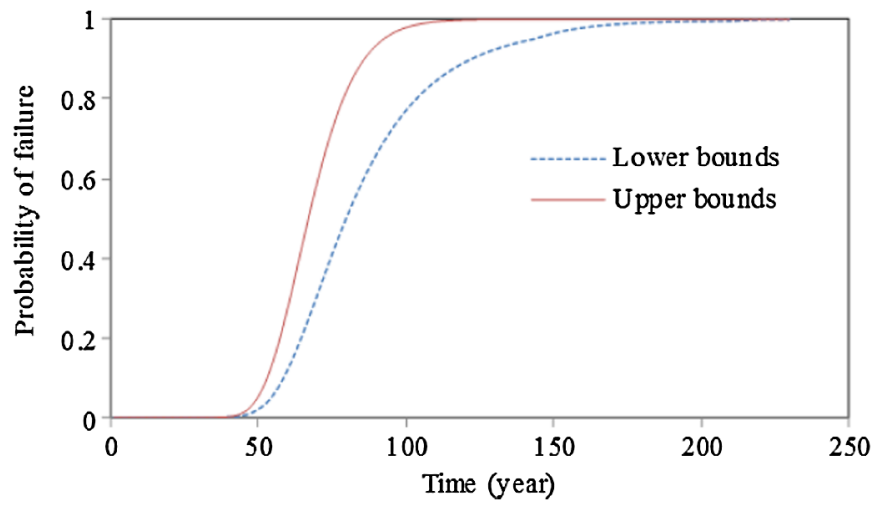

Fig. 6. Probability of failure for pipeline Section F using SS

optimization, for which 500 numbers of samples, level probability of 0.1 , and stopover value of $10^{-5}$ are used in this paper. The first column of Table 8 shows the optimal LCC cost, which is associated with the first maintenance.

Next, the proposed maintenance strategy is extended to determine an applicable and feasible renewal method using Tables 2 and 3. The recorded database shows that the underground pipelines are built on clay, sand, and sandy gravel. In addition, the whole pipeline network is above the groundwater level. Based on this information and according to Table 2, the possibility of soil loss for sanitary underground pipeline Sections A and B is low, whereas for 
Table 8. Results of Pipeline Risk-Cost Optimization Using SS

\begin{tabular}{lccclc}
\hline $\begin{array}{l}\text { Pipe } \\
\text { section }\end{array}$ & $\begin{array}{c}\text { Optimal life-cycle } \\
\text { cost [billion pounds }(£ b)]\end{array}$ & $\begin{array}{c}\text { Renewal } \\
\text { time (year) }\end{array}$ & $\begin{array}{c}\text { Structural condition } \\
\text { index }(C I)\end{array}$ & Renewal priority & Renewal methodology \\
\hline A & 2.2 & 60 & 1.9 & Low, minimal structural risk & Nonstructural or semistructural \\
B & 1.3 & 61 & 2.1 & Low, minimal structural risk & Nonstructural or semistructural \\
C & 1.34 & 67 & 2.3 & Low, minimal structural risk & Nonstructural or semistructural \\
D & 2.1 & 59 & 2.0 & Medium, poor condition & Semistructural, structural, or replacement \\
E & 1.4 & 71 & 2.5 & Medium, poor condition & Semistructural, structural, or replacement \\
F & 1.39 & 86 & 3.2 & Immediate, high structural risk & Structural or replacement \\
\hline
\end{tabular}

Table 9. Comparison between SS and GA Results

\begin{tabular}{|c|c|c|c|c|c|c|}
\hline \multirow[b]{2}{*}{$\begin{array}{l}\text { Pipe } \\
\text { section }\end{array}$} & \multicolumn{3}{|c|}{ SS method } & \multicolumn{3}{|c|}{ GA method } \\
\hline & $\begin{array}{c}\text { Optimal } \\
\text { life-cycle cost }(£ b)\end{array}$ & $\begin{array}{c}\text { Renewal } \\
\text { time (year) }\end{array}$ & $\begin{array}{c}\text { Structural } \\
\text { condition index }(C I)\end{array}$ & $\begin{array}{c}\text { Optimal } \\
\text { life-cycle cost }(£ b)\end{array}$ & $\begin{array}{c}\text { Renewal } \\
\text { time (year) }\end{array}$ & $\begin{array}{c}\text { Structural } \\
\text { condition index }(C I)\end{array}$ \\
\hline A & 2.2 & 60 & 1.9 & 2.41 & 62 & 2.2 \\
\hline B & 1.3 & 61 & 2.1 & 1.44 & 63 & 2.3 \\
\hline $\mathrm{C}$ & 1.34 & 67 & 2.3 & 1.42 & 66 & 2.25 \\
\hline $\mathrm{D}$ & 2.1 & 59 & 2.0 & 2.35 & 62 & 2.2 \\
\hline E & 1.4 & 71 & 2.5 & 1.32 & 72 & 2.5 \\
\hline $\mathrm{F}$ & 1.39 & 86 & 3.2 & 1.37 & 88 & 3.5 \\
\hline
\end{tabular}

Sections $\mathrm{C}$ to $\mathrm{F}$, the possibility of surrounding soil loss is high. The condition index $(C I)$ for the underground pipeline network is estimated as shown in Table 8 using Eq. (17) by substituting the identified optimal time to renew from risk-cost optimization. Applicable renewal categories are then selected from Table 3 based on $C I$ and the possible scenario of soil loss. Pipeline Sections A, B, and $\mathrm{C}$ are required to renew using nonstructural or semistructural lining methods based on the estimated $C I$ and low possibility of soil loss. On the other hand, due to high possibility of soil loss and $C I>2$, pipeline Sections $\mathrm{D}$ and $\mathrm{E}$ need to renew using semistructural or structural liners. Finally, pipeline Section F should be renewed with structural liners or replacement $(C I>3$, high possibility of soil loss). Alternatively, replacement is recommended if the repair cost becomes greater than the cost of replacing the pipes.

Based on the underground pipeline's inventory information and alignment, the renewal assessment has been carried out considering all six major impact factors and Eq. (16), and the renewal priority is based on the structural $C I$ and failure impact rating. According to Table 8, the pipes that are in fair or minimal structural risk condition need low renewal priority. On the other hand, pipes with a high structural risk condition require immediate rehabilitation or replacement for the safety of the network.

Finally, a comparison is made with GA results to validate the accuracy of the SS optimization process. Table 9 shows a good agreement between these two optimization approaches. The obtained optimal costs and renewal times from SS and GA methods are reasonably close. The renewal time and renewal methodologies are the same for both optimization approaches. The advantage of SS optimization is that it takes about $30 \mathrm{~min}$ to execute the current problem, whereas GA optimization takes more than $1 \mathrm{~h} 30 \mathrm{~min}$. Thus, SS requires only one-third the computational time compared to GA. Note that the computational speed is measured in terms of central processing unit (CPU) time on a 1.6-GHz Pentium IV personal computer in this paper. Another advantage of SS optimization is that the evaluation number required by GA is larger than that of the SS algorithm. The SS algorithm exhibits a higher success ratio compared to GA and enables finding the global maxima in all runs.
Thus, SS is found to be the best choice for this kind of multimodal function.

\section{Conclusions}

An SS optimization technique has been developed in this paper for reliability-based risk-cost optimization of a buried pipeline network. The proposed optimization technique is found to be competitive in exploiting the feasible regions and providing optimal solutions in solving complex problems. A numerical example is presented to demonstrate the robustness and validate the efficiency of the technique. SS is first employed to estimate the reliability of flexible underground pipes subjected to externally applied loading and material corrosion. Then SS is extended to determine the intervention year for maintenance and to identify the most appropriate renewal solution and renewal priority by minimizing the risk of failure and whole life-cycle cost. A comparison is made with the results obtained from GA to validate the accuracy of the SS optimization. The paper shows a good agreement between these two methods. However, SS requires only one-third the computational time of GA. The proposed risk-cost optimization approach can help management in making correct decisions concerning the intervention year and renewal methodology. The proposed method produces a reasonably good result with much less computational effort.

\section{References}

Ahammed, M., and Melchers, R. E. (1997). "Probabilistic analysis of pipelines subject to combined stresses and corrosion." Eng. Struct., 19(12), 988-994.

Alani, A. M., Faramarzi, A., Mahmoodian, M., and Tee, K. F. (2014). "Prediction of sulphide build-up in filled sewer pipes." Environ. Technol., 35(14), 1721-1728.

Ambrose, M. D., Burn, S., Desilva, D., and Rahilly, M. (2008). "Life cycle analysis of water networks." XIV Plastics Pipes Conf., Plastic Pipes Conference Association, Budapest, Hungary. 
Au, S. K., and Beck, J. L. (2001). "Estimation of small failure probabilities in high dimensions by subset simulation." Probab. Eng. Mech., 16(4), 263-277.

Au, S. K., and Beck, J. L. (2003). "Subset simulation and its application to seismic risk based on dynamic analysis." J. Eng. Mech., 10.1061/ (ASCE)0733-9399(2003)129:8(901), 901-917.

Au, S. K., Ching, J., and Beck, J. L. (2007). "Application of subset simulation methods to reliability benchmark problems." Struct. Saf., 29(3), 183-193.

AWWA (American Water Works Association). (1999). Buried pipe design, fiberglass pipe design, Denver.

Beaurepaire, P., Valdebenito, M. A., Schueller, G. I., and Jensen, H. A. (2012). "Reliability-based optimization of maintenance scheduling of mechanical components under fatigue." Comput. Methods Appl. Mech. Eng., 221-222, 24-40.

Chen, H. M., Hou, C. C., and Wang, Y. H. (2013). "A 3D visualized expert system for maintenance and management of existing building facilities using reliability-based method." Expert Syst. Appl., 40(1), 287-299.

Ching, J., Beck, J. L., and Au, S. K. (2005). "Hybrid subset simulation method for reliability estimation of dynamical systems subject to stochastic excitement." Probab. Eng. Mech., 20(3), 199-214.

Davis, P., De Silva, D., Marlow, D., Moglia, M., Gould, S., and Burn, S. (2008). "Failure prediction and optimal scheduling of replacements in asbestos cement water pipes." J. Water Supply: Res. Technol., 57(4), 239-252.

Enright, M. P., and Frangopol, D. M. (1998). "Service-life prediction of deteriorating concrete bridges." J. Struct. Eng., 10.1061/(ASCE) 0733-9445(1998)124:3(309), 309-317.

Fang, Y., Chen, J., and Tee, K. F. (2013). "Analysis of structural dynamic reliability based on the probability density evolution method." Struct. Eng. Mech., 45(2), 201-209.

Fang, Y., Wen, L., and Tee, K. F. (2014). "Reliability analysis of repairable k-out-n system from time response under several times stochastic shocks." Smart Struct. Syst., 14(4), 559-567.

Fetz, T., and Tonon, F. (2008). "Probability bounds for series systems with variables constrained by sets of probability measures." Int. J. Reliab. Saf., 2(4), 309-339.

Gabriel, L. H. (2011). Corrugated polyethylene pipe design manual and installation guide, Plastic Pipe Institute, Irving, TX.

Halfawy, M. R., Dridi, L., and Baker, S. (2008). "Integrated decision support system for optimal renewal planning of networks." J. Comput. Civ. Eng., 10.1061/(ASCE)0887-3801(2008)22:6(360), 360-372.

Hancor. (2009). "High density polyethylene (HDPE) pipe design." Chapter 2, Drainage handbook, Findlay, $\mathrm{OH}, 5-21$.

Hinow, M., Waldron, M., Müller, L., Aeschbach, H., and Pohlink, K. (2008). "Substation life cycle cost management supported by stochastic optimization algorithm." 42nd Int. Council on Large Electric Systems, International Council on Large Electric Systems, Paris, B3-103.

Khan, L. R., Tee, K. F., and Alani, A. M. (2013). "Reliability-based management of underground pipeline network using genetic algorithm." Proc., 11th Int. Probabilistic Workshop, Brno Univ. of Technology, Brno, Czech Republic, 159-171.

Li, H. (2011). "Subset simulation for unconstrained global optimization." J. Appl. Math. Modell., 35(10), 5108-5120.

Li, H., and Au, S. K. (2010). "Design optimization using subset simulation algorithm." Struct. Saf., 32(6), 384-392.

Lounis, Z. (2006). "Risk-based maintenance optimization of aging highway bridge decks." Adv. Eng. Struct., Mech. Constr., 140, 723-734.

Mahmoodian, M., Alani, A. M., and Tee, K. F. (2012). "Stochastic failure analysis of the gusset plates in the Mississippi River bridge." Int. J. Forensic Eng., 1(2), 153-166.

McDonald, S., and Zhao, J. (2001). "Condition assessment and rehabilitation of large sewer." Int. Conf. on Underground Infrastructure Research, Univ. of Waterloo, Waterloo, Canada, 361-369.
Melbourne Water. (2012). Melbourne main sewer replacement, Melbourne, Australia.

Moan, T. (2005). "Reliability-based management of inspection, maintenance and repair of offshore structures." Struct. Infrastruct. Eng., $1(1), 33-62$.

Newton, L. A., and Vanier, D. J. (2006). "MIIP report: The state of Canadian sewer-Analysis of asset inventory and condition." National Research Council, Institution for Research in Construction, Ottawa.

Rahman, S., and Vanier, D. J. (2004). "Life cycle cost analysis as a decision support tool for managing municipal infrastructure." Int. Council for Research and Innovation in Building and Construction (CIB 2004) Triennial Congress, Delft, the Netherlands.

Ravindran, A., Ragsdell, K. M., and Reklaitis, G. V. (2006). Engineering optimization: Methods and application, 2nd Ed., Wiley, NJ.

Sadiq, R., Rajani, B., and Kleiner, Y. (2004). "Probabilistic risk analysis of corrosion associated failures in cast iron water mains." Reliab. Eng. Syst. Saf., 86(1), 1-10.

Saydam, D., and Frangopol, D. M. (2015). "Risk-based maintenance optimization of deteriorating bridges." J. Struct. Eng., 10.1061/(ASCE)ST .1943-541X.0001038, 04014120.

Schueller, G. I., and Pradlwarter, H. J. (2007). "Benchmark study on reliability estimation in higher dimensions of structural systems-An overview." Struct. Saf., 29(3), 167-182.

Sivakumar Babu, G. L., and Rao, R. S. (2005). "Reliability measures for buried flexible pipes." Can. Geotech. J., 42(2), 541-549.

Sivakumar Babu, G. L., and Srivastava, A. (2010). "Reliability analysis of buried flexible pipe-soil systems." J. Pipeline Syst. Eng. Pract., 10 .1061/(ASCE)PS.1949-1204.0000041, 33-41.

Spall, J. C. (2003). Introduction to stochastic search and optimization: Estimation, simulation, and control, Wiley, Hoboken, NJ.

Tee, K. F., and Khan, L. R. (2012). "Risk-cost optimization and reliability analysis of underground pipelines." Proc., 6th Int. ASRANet Conf., London.

Tee, K. F., and Khan, L. R. (2014). "Reliability analysis of underground pipelines with correlation between failure modes and random variables." J. Risk Reliab., Proc., Inst. Mech. Eng., Part O, 228(4), 362-370.

Tee, K. F., Khan, L. R., and Chen, H. P. (2013). "Probabilistic failure analysis of underground flexible pipes." Struct. Eng. Mech., 47(2), $167-183$

Tee, K. F., Khan, L. R., Chen, H. P., and Alani, A. M. (2014a). "Reliability based life cycle cost optimization for underground pipeline networks." Tunnelling Underground Space Technol., 43, 32-40.

Tee, K. F., Khan, L. R., and Coolen-Maturi, T. (2015). "Application of receiver operating characteristic curve for pipeline reliability analysis." J. Risk Reliab., Proc. Inst. Mech. Eng., Part O, 229(3), 181-192.

Tee, K. F., Khan, L. R., and Li, H. (2014b). "Application of subset simulation in reliability estimation of underground pipelines." Reliab. Eng. Syst. Saf., 130, 125-131.

Tee, K. F., and Li, C. Q. (2011). "A numerical study of maintenance strategy for concrete structures in marine environment." Proc., 11th Int. Conf. on Applications of Statistics and Probability in Civil Engineering, CRC Press, London, 618-625.

Tee, K. F., Li, C. Q., and Mahmoodian, M. (2011). "Prediction of time-variant probability of failure for concrete pipes." 12th Int. Conf. on Durability of Building Materials and Components, Univ. of Porto, Portugal.

Tolentino, D., and Ruiz, S. E. (2013). "Time intervals for maintenance of offshore structures based on multiobjective optimization." Math. Prob. Eng., 15.

Watkins, R. K., and Anderson, L. R. (2000). Structural mechanics of buried pipes, CRC Press, Washington, DC.

WRC (Water Research Centre). (2001). Sewerage rehabilitation manual, 4th Ed., Wiltshire, U.K. 